\title{
Instability and Spatiotemporal Dynamics of Alternans in Paced Cardiac Tissue
}

\author{
Blas Echebarria and Alain Karma \\ Department of Physics and Center for Interdisciplinary Research on Complex Systems, Northeastern University, \\ Boston, Massachusetts 02115
}

(Received 28 November 2001; published 6 May 2002)

\begin{abstract}
We derive an equation that governs the spatiotemporal dynamics of small amplitude alternans in paced cardiac tissue. We show that a pattern-forming linear instability leads to the spontaneous formation of stationary or traveling waves whose nodes divide the tissue into regions with opposite phase of oscillation of action potential duration. This instability is important because it creates dynamically a heterogeneous electrical substrate for the formation of conduction blocks and the induction of fibrillation if the tissue size exceeds a fraction of the pattern wavelength. We derive an analytical expression for this wavelength as a function of three basic length scales related to dispersion and intercellular electrical coupling.
\end{abstract}

DOI: $10.1103 /$ PhysRevLett.88.208101

It is well established that the duration of cardiac excitation can oscillate from beat to beat at sufficiently short pacing interval [1]. Pioneering studies by Nolasco and Dahlen [2] and Guevara et al. [3] have demonstrated that the generic sequence $L S L S \ldots$ of long and short action potential duration (APD), known as alternans, is a direct consequence of the restitution relationship

$$
\mathrm{APD}^{n+1}=f\left(\mathrm{DI}^{n}\right)
$$

between the APD generated by the $n$th +1 stimulus, $\mathrm{APD}^{n+1}$, and the diastolic time interval $\mathrm{DI}^{n}$ during which the tissue recovers its resting properties after the end of the previous ( $n$ th) action potential. If we denote the interval between the $n$th and $n$th +1 stimulus by $T^{n}$, we must have $\mathrm{DI}^{n}=T^{n}-\mathrm{APD}^{n}$. Then, for a fixed period: $T^{n}=\tau$ for all $n$, Eq. (1) yields the map $\mathrm{APD}^{n}=$ $f\left(\tau-\mathrm{APD}^{n-1}\right)$, whose slope $f^{\prime}$ typically increases with decreasing period. If the slope of the restitution curve exceeds unity, the map undergoes a period doubling bifurcation to alternans.

Over the last decade, the study of alternans [4-11], and their control [12], has become a main focus of research because of the potentially crucial link of this dynamical instability with cardiac fibrillation [13]. However, there is presently no simple analytical understanding of how the bifurcation to alternans is manifested spatiotemporally in paced cardiac tissue. Analytical progress to date is limited to the one-dimensional circulation of electrical impulse in a ring of tissue [5-7].

In this Letter, we derive an equation that governs the spatiotemporal dynamics of alternans close to the onset of instability. This enables us to obtain a quantitative analytical understanding of the formation of recently observed complex patterns of APD oscillations that can promote fibrillation [8-11]. A crucial feature of these patterns is that the APD oscillates with opposite phases in two (or more) spatially extended regions of tissue, i.e., with a sequence $L S L S \ldots$ in one region and $S L S L \ldots$ in the other. These "discordant alternans" have been observed experimentally in both two-dimensional [8] and linear strands [9] of car-
PACS numbers: 87.19.Hh, 05.45.-a, 05.45.Gg, 89.75.-k

diac tissue, as well as in ionic model simulations [9-11]. Moreover, they have been shown to lead to the formation of conduction blocks [9] as well as to the onset of spiral wave formation and fibrillation [8]. We show here that discordant alternans result from a pattern-forming linear instability that has interesting similarities with classic instabilities leading to the spontaneous formation of spatially periodic patterns in nature (such as Rayleigh-Bénard convection, Taylor-Couette flow, etc. [14]), but also presents some unique features.

We consider a one-dimensional (1D) homogeneous cable of length $L$ paced at period $\tau$ from one end $(x=0)$. Close to the onset of instability, we can expand the APD and the period in the form

$$
\begin{aligned}
& \operatorname{APD}^{n}(x) \approx \operatorname{APD}_{c}+a(x, t) e^{i \pi n}, \\
& T^{n}(x) \approx \tau_{c}-\delta \tau+b(x, t) e^{i \pi n},
\end{aligned}
$$

where $\mathrm{APD}_{c}$ and $\tau_{c}$ are the APD and the period evaluated at the bifurcation point of the map $\left(f^{\prime}=1\right), x$ measures the position along the cable, and $\delta \tau \equiv \tau_{c}-\tau \ll \tau_{c}$. In this range of period, $a$ and $b$ vary slowly from beat to beat, which allows us to treat the time, $t \equiv n \tau$, as a continuous variable; the fast beat-to-beat oscillations are contained in the exponential factor $e^{i \pi n}$.

A relation between $a$ and $b$ can first be derived by noting that $T^{n}(x)$ is the difference of arrival time of two subsequent action potentials at $x$ [11], or

$$
T^{n}(x)=\tau+\int_{0}^{x} \frac{d x^{\prime}}{c\left(\mathrm{DI}^{n}\left(x^{\prime}\right)\right)}-\int_{0}^{x} \frac{d x^{\prime}}{c\left(\mathrm{DI}^{n-1}\left(x^{\prime}\right)\right)},
$$

where the first (second) integral on the right-hand side is the time required for the leading front of the action potential to travel from the paced end to $x$ at the $n$th $(n$th -1$)$ stimulus; concomitantly, $c(\mathrm{DI})$ is the standard dispersion curve that relates the propagation speed of this front with the local diastolic interval. The dispersion curve is typically steeply increasing at small DI and flat at large DI. Substituting Eqs. (2), (3) into Eq. (4) with $\mathrm{DI}^{n}(x)=$ $T^{n}(x)-\operatorname{APD}^{n}(x)$, and expanding to linear order in $a$ and $b$, we obtain $b(x) \simeq \int_{0}^{x} a\left(x^{\prime}\right) d x^{\prime} / \Lambda$ where we have 
defined $\Lambda \equiv c^{2} / 2 c^{\prime}$, with $c$ and $c^{\prime} \equiv d c / d$ DI evaluated at the bifurcation point, and assumed that $\Lambda$ is much larger than the scale over which $a$ varies.

Next, in order to derive an evolution equation for the amplitude $a(x, t)$, we first neglect the influence of the electrical coupling between cells on the APD. This allows us to assume that the restitution relationship (1), and hence the second iteration of the map

$$
\mathrm{APD}^{n+2}=f\left(T^{n+1}-f\left(T^{n}-\mathrm{APD}^{n}\right)\right),
$$

continues to be valid even when the APD is nonspatially uniform; we shall soon see why it is crucial to relax this assumption. We substitute Eqs. (2),(3) into Eq. (5), and expand the right-hand side keeping only the dominant linear and weakly nonlinear terms. Furthermore, we use the aforementioned fact that, close to onset, $a$ varies slowly from beat to beat, and we therefore expand the left-hand side as $\mathrm{APD}^{n+2} \simeq \mathrm{APD}^{n}+2 \partial a / \partial n e^{i \pi n}$, where $\partial a / \partial n=\tau \partial a / \partial t$. Finally, we use the integral relation between $a$ and $b$ derived earlier. After equating both sides of Eq. (5), we obtain

$$
\tau \partial_{t} a=\sigma a-g a^{3}-\int_{0}^{x} \frac{d x^{\prime}}{\Lambda} a\left(x^{\prime}\right),
$$

where $\sigma \equiv f^{\prime \prime}\left(\tau-\tau_{c}\right) / 2, g \equiv f^{\prime \prime 2} / 4-f^{\prime \prime \prime} / 6$, and all derivatives are evaluated at the bifurcation point.

In order to test this evolution equation, we simulate the standard cable equation

$$
\partial_{t} V=D \partial_{x}^{2} V-\left(I_{\text {ion }}+I_{\text {ext }}\right) / C_{m}
$$

for the membrane current $I_{\text {ion }}$ given by the Noble model [15] with time in units of millisecond (ms), $D=2.5 \times$ $10^{-4} \mathrm{~cm}^{2} / \mathrm{ms}, C_{m}=12 \mu \mathrm{F} / \mathrm{cm}^{2}, d x=0.01 \mathrm{~cm}, d t=$ $0.05 \mathrm{~ms}$, and $I_{\mathrm{ext}}$ modeling a sequence of stimuli applied at $x=0$ at the pacing interval $\tau$. To determine the parameters of the amplitude equation, we compute the restitution and dispersion curves by pacing Eq. (7) in a short cable and by using two subsequent stimuli spaced by different intervals to vary DI; we also use $V=-40 \mathrm{mV}$ as a threshold of the transmembrane voltage to define the APD. We impose zero gradient boundary conditions on $V$ and $a$ at the two ends of the cable in all our simulations.

Figure 1(a) shows that Eq. (6) produces discordant alternans consistent with the picture that restitution and dispersion suffice to produce this state [9-11]. However, the magnitude of the spatial gradient of $a$ at the node increases with time, and can be shown to diverge in a finite time. This yields an unphysical spatial discontinuity of APD, which also occurs if the system of coupled maps [Eqs. (1) and (4)], from which the amplitude equation is derived, is solved numerically. Note that the analogous system of coupled maps for a 1D pulse circulating in a ring produces a smooth APD modulation starting from a smooth initial condition [5], which highlights the much stronger role of dispersion at producing heterogeneity of APD during pacing than circulation.

This discontinuity, which is absent in the simulation of the cable equation in Fig. 1(b), can be cured by adding

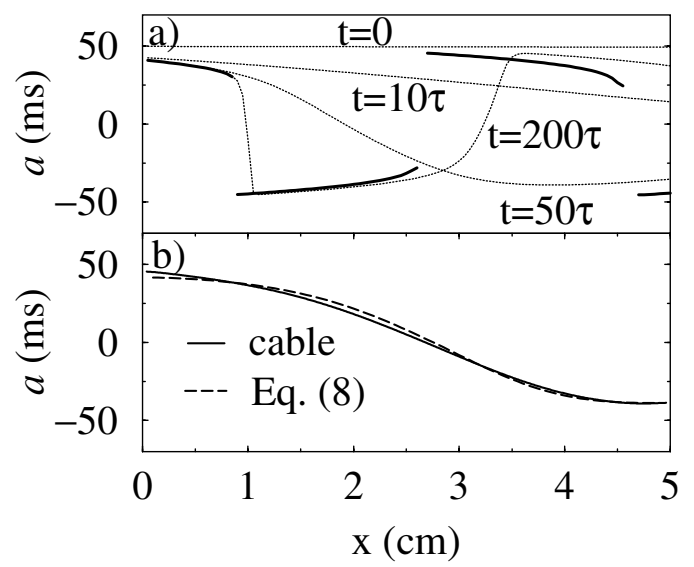

FIG. 1. Amplitude $a$ of APD oscillation vs $x$ for Noble parameters and $\tau=258 \mathrm{~ms}$. (a): profiles obtained with Eq. (6) at different times (dotted lines) and final stationary profile (solid line). (b): stationary profiles obtained with Eq. (7) (solid line) and Eq. (8) (dashed line). Nodes $(a=0)$ separate tissue regions with $\pi$ out of phase oscillations.

spatial derivative terms to the amplitude equation. Since the cable is paced at one end, the underlying basic state (i.e., traveling pulses) is not invariant under parity symmetry. Hence, in addition to $\partial_{x}^{2} a$, a term proportional to $\partial_{x} a$ must generally be included, which yields the final form of our amplitude equation

$$
\tau \partial_{t} a=\sigma a-g a^{3}-\int_{0}^{x} \frac{d x^{\prime}}{\Lambda} a\left(x^{\prime}\right)-w \partial_{x} a+\xi^{2} \partial_{x}^{2} a .
$$

There is a close analogy between this equation and the real Ginzburg-Landau equation that has been extensively studied in the context of phase transitions and front propagation [14]. The dynamics is richer here because the integral term originating from dispersion causes a nonlocal interaction of the fronts separating two out-of-phase oscillating regions with the pacing end of the cable.

To calculate the new length scales $w$ and $\xi$, we must determine how the electrical coupling between cells modifies the restitution relationship [Eq. (1)]. For complex electrophysiological models such as Noble, this generally needs to be done numerically using a procedure that will be discussed elsewhere. For a simple two-variable ionic model, which we study below, the analytical expressions

$$
\begin{gathered}
w=2 D / c, \\
\xi=\left(D \times A P D_{c}\right)^{1 / 2},
\end{gathered}
$$

can be derived by interpreting Eq. (7) as a diffusion equation with a source $I_{\text {ion }}$, and expressing $V$ as space-time integral of $G I_{\text {ion }}$, where $G$ is the standard Green's function of the diffusion equation. This integral can then be evaluated analytically because the action potential shape is simply triangular for this model, and used to calculate $w$ and $\xi$. Equation (10) has the simple physical interpretation that $V$ diffuses a length $\sim \xi$ in the time interval of one APD. Therefore, the repolarization of a given cell is influenced by other cells within a length $\sim \xi$ of cable. In 
addition, repolarization of this cell is influenced unequally by its left and right neighboring cells because these cells are activated at different times by the propagating wave front. Clearly, this asymmetry must vanish in the limit $c \rightarrow \infty$ where all cells are activated simultaneously consistent with Eq. (9). Figure 1(b) shows that our regularized amplitude equation (8) now produces a smoothly varying stationary profile of $a$ that agrees well with the simulation of the cable-Noble equation, where $a$ is obtained from the APD using Eq. (2).

The genesis of discordant alternans can be understood by computing the linear stability spectrum of the spatially homogeneous state $(a=0)$. We have calculated this spectrum both numerically for different $L$, and analytically for the large $L$ limit. The main result is that the wave pattern can emerge from the amplification of either a unique finite wavelength mode, which yields a stationary pattern, or from a discrete set of complex modes that approach a continuum in the limit $L \rightarrow \infty$, and yields a traveling pattern. There is indeed experimental evidence for both stationary [8] and traveling [9] waves.

We treat here explicitly the large $L$ limit since it provides the basis to understand finite- $L$ patterns. In this limit, we can analyze stability by differentiating Eq. (8) with respect to $x$ and letting $a(x, t) \sim e^{i k x+\Omega t / \tau}$, with both $\Omega \equiv \Omega_{r}+$ $i \Omega_{i}$ and $k \equiv k_{r}+i k_{i}$ complex, which yields at once the eigenvalue equation

$$
\Omega=\sigma-\xi^{2} k^{2}-i[w k-1 /(\Lambda k)] .
$$

When dispersion is weak, a unique real mode with $k_{i}=0$ grows faster than the other complex modes and yields a stationary pattern. Its wavelength $\lambda=2 \pi / k_{r}$, is determined by the condition $\Omega_{i}=0$, which yields

$$
\lambda=2 \pi(w \Lambda)^{1 / 2},
$$

in good agreement with the wavelength observed in simulations of the cable-Noble equation (Table I). Note that $\cos k_{r} x$ is an exact eigenvector of Eq. (8) linearized around $a=0$ that satisfies $\partial_{x} a=0$ at the two cable ends when $L$ is an integer multiple of $\lambda / 2$. The threshold of instability occurs when $\Omega_{r}=0$, or for a period $\tau_{\text {th }}$ defined by $\sigma_{\text {th }}=$ $f^{\prime \prime}\left(\tau_{\text {th }}-\tau_{c}\right) / 2=\xi^{2} / w \Lambda$.

In the opposite limit where dispersion is strong, complex modes that grow exponentially at large $x$ are the most unstable. This exponential growth is responsible for the formation of conduction blocks at the end of the cable opposite to the pacing site, as recently seen experimentally [9]. It is simple to deduce from Eq. (11) that each $k$ mode travels towards the pacing end of the cable, but a

TABLE I. Values of various lengths in $\mathrm{cm}$ with $\lambda_{\text {theor }}$ [Eqs. (12) or (13)] and $\lambda_{\text {sim }}$ [from simulations of Eq. (7)].

\begin{tabular}{lcccccc}
\hline \multicolumn{1}{c}{ Model } & $\Lambda$ & $\mathrm{w}$ & \multicolumn{1}{c}{$\xi$} & $\lambda_{\text {theor }} / 4$ & $\lambda_{\text {sim }} / 4$ & $L_{\text {min }}$ \\
\hline Noble & 49.1 & 0.045 & 0.18 & 2.33 & 2.6 & 2.75 \\
Two-variable & 3.55 & 0.031 & 0.235 & 1.33 & 1.1 & 1.15 \\
\hline \hline
\end{tabular}

208101-3 wave packet constructed from a linear superposition of these modes has a group velocity that makes the packet move away from the pacing end. This is the signature of a convective instability [14] where perturbations are transported as they grow, similarly to, for example, TaylorCouette vortices developing in an axial flow [16]. In such a situation, patterns are only transient unless the group velocity vanishes, or $\partial \Omega_{i} / \partial k_{r}=0$, and hence they grow at a fixed point in space. Moreover, the fastest growing wavelength that dominates at large time must correspond to a maximum of $\Omega_{r}$, which yields the additional condition $\partial \Omega_{r} / \partial k_{r}=0$. From this, we deduce that the threshold of absolute instability occurs when $\sigma_{\text {th }}=(3 / 2)(\xi / 2 \Lambda)^{2 / 3}$, with a pattern of wavelength

$$
\lambda=(4 \pi / \sqrt{3})\left(2 \xi^{2} \Lambda\right)^{1 / 3},
$$

which travels with phase velocity $\Omega_{i} \lambda /(2 \pi)$ where $\Omega_{i}=$ $(3 \sqrt{3} / 2)(\xi / 2 \Lambda)^{2 / 3}$.

Traveling waves are favored over a stationary pattern when $\Lambda=2 c^{2} / c^{\prime} \ll \xi^{4} / w^{3}$, and hence for large enough dispersion $\left(c^{\prime}\right)$, and vice versa in the opposite limit. The APD oscillations at a fixed $x$ are periodic when the wave is stationary and quasiperiodic when it travels. For both cases, we find here that $\lambda$ is independent of $L$, in contrast to the oscillations produced by a pulse circulating in a ring where it is known that $\lambda \simeq 2 L /(2 i+1)$ for weak dispersion [5] (with $i$ integer and $L=$ ring perimeter). As will be discussed elsewhere, our theory applied to the ring shows that the bifurcation to alternans is finite dimensional with $i=0$ being the most unstable mode, in agreement with the fact that it is this mode that is generically selected in experiments [4] and ionic model simulations [5-7]. In addition, it shows that the gradient term $\left(-w \partial_{x} a\right)$ can lead to quasiperiodicity even in the absence of dispersion $\left(c^{\prime}=0\right)$.

As a final test of our theory, we study a two-variable model where all the parameters of the amplitude equation can be calculated analytically including $w$ and $\xi$ given by Eqs. (9) and (10), and for which our theory predicts traveling waves. In this model, $I_{\text {ion }} / C_{m}$ is the sum of a slow outward current, $I_{o} / C_{m}=\tau_{0}^{-1}\left[S+(1-S) V / V_{c}\right]$, and a fast inward current, $I_{i} / C_{m}=-\tau_{a}^{-1} h S$, where inactivation of the latter is controlled by

$$
\partial_{t} h=(1-S-h) /\left[\tau^{-}(1-S)+S \tau^{+}\right] .
$$

In addition, $V$ is dimensionless and $S \equiv\{1+\tanh [(V-$ $\left.\left.\left.V_{c}\right) / \epsilon\right]\right\} / 2$. We choose $V_{c}=0.1, \tau_{0}=150 \mathrm{~ms}, \tau_{a}=$ $6 \mathrm{~ms}, \tau^{-}=60 \mathrm{~ms}, \tau^{+}=12 \mathrm{~ms}, \epsilon=0.005$, and we simulate Eq. (7) with the same $D$ as before, $d x=$ $0.01 \mathrm{~cm}, d t=0.02 \mathrm{~ms}$, and $V=0.1$ as threshold for the APD.

As predicted, we observe traveling waves in this model with a wavelength that agrees well with Eq. (13). Figure 2 illustrates sustained and transient wave patterns above (left) and below (right) the onset of absolute instability, respectively. Figure 3 shows a good agreement between the results of simulations of the cable and amplitude 


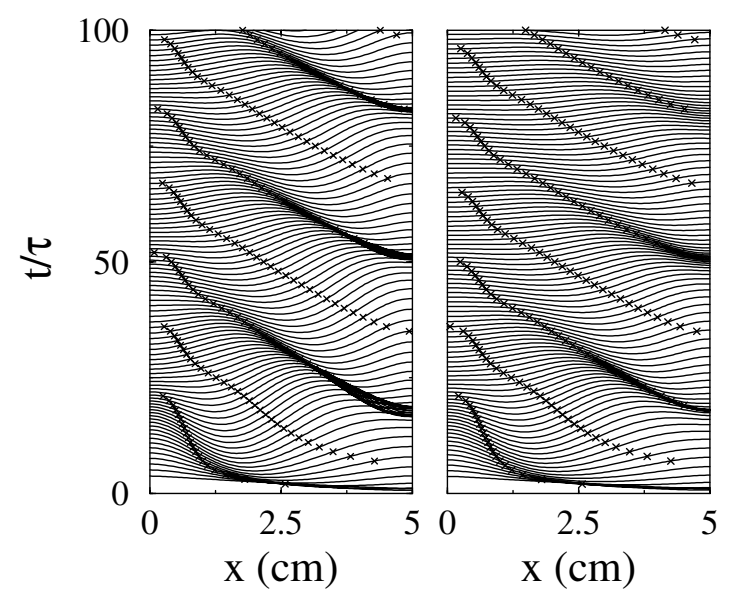

FIG. 2. Space-time plots of $a$ obtained by simulations of Eq. (8) for parameters of the two-variable model, showing absolutely unstable (left and $\tau=295 \mathrm{~ms}$ ) and convectively unstable (right and $\tau=298 \mathrm{~ms}$ ) wave patterns. The crosses denote the positions of the nodes $(a=0)$.

equations. It confirms the analytical prediction that the onset of instability occurs at a shorter period in a larger cable, and hence for a slope of the restitution curve larger than unity. Figure 3 is qualitatively similar for stationary waves in the Noble model, but this overstabilization is smaller because dispersion is weaker.

Our results demonstrate that the formation of discordant alternans is crucially affected by the effect of electrical coupling (diffusion) on repolarization, in addition to restitution and dispersion. Dispersion is responsible for the formation of nodes and spatial gradients of APD that steepen with time. Diffusion, in turn, tends to spread the APD spatially, and also induces a drift of the pattern away from the pacing site caused by the more subtle gradient term $\left(-w \partial_{x} a\right)$ in the amplitude equation. When dispersion is sufficiently weak, drift balances dispersion and produces a stationary pattern. In the opposite limit, the tendency for dispersion to form steep gradients of APD is balanced by the spreading effect of diffusion. Nodes then travel, cyclically disappearing (appearing) at the pacing (opposite) end of the cable.

In conclusion, we have derived a simple evolution equation that describes the universal spatiotemporal dynamics of small amplitude alternans in paced cardiac tissue. We have shown that discordant wave patterns [8-11], which are linked to fibrillation [8], result from a finite wavelength linear instability. Hence, their formation requires a minimum tissue size $L_{\min } \sim \lambda / 4$, required for at least one node to form. The value of $L_{\min }$ that we measure in simulations of reaction-diffusion models is actually close to $\lambda / 4$ with $\lambda$ predicted by Eqs. (12) and (13), respectively (Table I). This equation can be generalized to higher dimensions as well as to include spatial gradients of electrical properties that influence the pattern evolution. In addition, its derivation can be extended to higher dimensional maps that
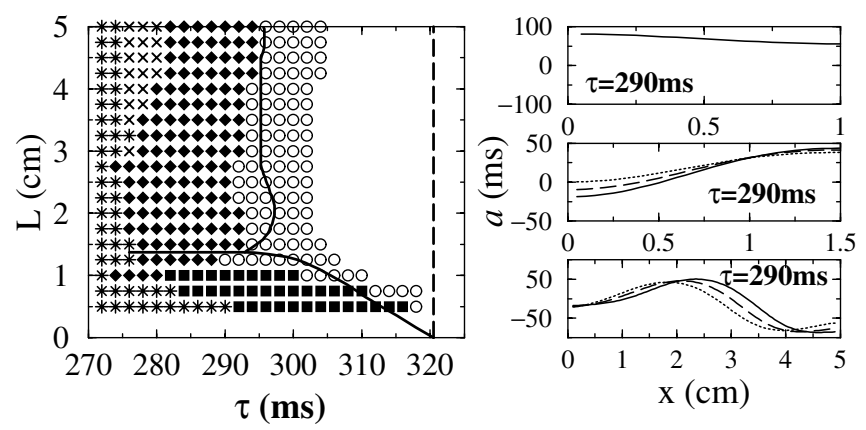

FIG. 3. Stability diagram of two-variable cable model with domains of no-alternans (open circles), concordant alternans (filled squares), discordant alternans (filled diamonds), and 2:1 conduction blocks at (stars) or away from (crosses) the pacing site. Boundaries between the same domains obtained by simulations of the amplitude equation (8) are shown by solid lines. The dashed line denotes the bifurcation period for alternans predicted by the map of Eq. (1). On the right panel we show profiles of $a$ vs $x$ at times $t$ (solid), $t+2 \tau$ (dashed), and $t+4 \tau$ (dotted lines), for three different cable lengths.

include memory effects and have a period doubling bifurcation. Finally, this equation is readily applicable to model a nonconstant pacing interval and provides a theoretical basis to study the control of alternans in spatially extended tissue.

This research was supported in part by NIH SCOR in Sudden Cardiac Death P50-HL52319.

[1] G. R. Mines, J. Physiol. (London) 46, 349 (1913).

[2] J. B. Nolasco and R. W. Dahlen, J. Appl. Physiol. 25, 191 (1968).

[3] M. R. Guevara et al., in Proceedings of the 11th Computers in Cardiology Conference (IEEE Computer Society, Los Angeles, 1984), p. 167.

[4] L. H. Frame and M.B. Simson, Circulation 78, 1277 (1988).

[5] M. Courtemanche, L. Glass, and J. P. Keener, Phys. Rev. Lett. 70, 2182 (1993).

[6] A. Karma, Chaos 4, 461 (1994).

[7] A. Vinet, Ann. Biomed. Eng. 28, 704 (2000).

[8] J. M. Pastore et al., Circulation 99, 1385 (1999).

[9] J. J. Fox et al., Circ. Res. 90, 289 (2002).

[10] Z. Qu et al., Circulation 102, 1664 (2000).

[11] M. A. Watanabe et al., J. Cardiovasc. Electrophys. 12, 196 (2001).

[12] K. Hall et al., Phys. Rev. Lett. 78, 4518 (1997); D. J. Christini et al., Proc. Nat. Acad. Sci. U.S.A. 98, 5827 (2001).

[13] See A. Karma, Proc. Nat. Acad. Sci. U.S.A. 97, 5687 (2000); D. S. Rosenbaum, J. Cardiovasc. Electrophys. 12, 207 (2001); and earlier references therein.

[14] M. C. Cross and P. C. Hohenberg, Rev. Mod. Phys. 65, 851 (1993).

[15] D. Noble, J. Physiol. 160, 317 (1962).

[16] K. L. Babcock, G. Ahlers, and D. S. Cannell, Phys. Rev. Lett. 67, 3388 (1991). 